\title{
Influence of the shape of the micro-finite element model on the mechanical properties calculated from micro-finite element analysis
}

\author{
XIN-XIN WEN $^{1 *}$, HAI-LONG YU ${ }^{2 *}$, YA-BO YAN ${ }^{3 *}$, CHUN-LIN ZONG ${ }^{4}$, \\ HAI-JIAO DING ${ }^{1}$, XIANG-YU MA ${ }^{1}$, TIAN-SHENG WANG ${ }^{1}$ and WEI LEI ${ }^{3}$ \\ ${ }^{1}$ Department of Orthopedics, 463 Hospital of PLA, Shenyang, Liaoning 110042; ${ }^{2}$ Department of Orthopedics, \\ General Hospital of Shenyang Military Area Command of PLA, Rescue Center of Severe Wound and \\ Trauma of PLA, Shenyang, Liaoning 110016; ${ }^{3}$ Department of Orthopedics, Xijing Hospital; \\ ${ }^{4}$ Department of Cranio-facial Trauma and Orthognathic Surgery, School of Stomatology, \\ Fourth Military Medical University, Xi'an, Shaanxi 710032, P.R. China
}

Received February 5, 2017; Accepted April 21, 2017

DOI: $10.3892 /$ etm.2017.4709

\begin{abstract}
Assessing the biomechanical properties of trabecular bone is of major biological and clinical significance for the research of bone diseases, fractures and their treatments. Micro-finite element ( $\mu \mathrm{FE}$ ) models are becoming increasingly popular for investigating the biomechanical properties of trabecular bone. The shapes of $\mu \mathrm{FE}$ models typically include cube and cylinder. Whether there are differences between cubic and cylindrical $\mu \mathrm{FE}$ models has not yet been studied. In the present study, cubic and cylindrical $\mu \mathrm{FE}$ models of human vertebral trabecular bone were constructed. A $1 \%$ strain was prescribed to the model along the superior-inferior direction. E values were calculated from these models, and paired t-tests were performed to determine whether these were any differences between $\mathrm{E}$ values obtained from cubic and cylindrical models. The results demonstrated that there were no statistically significant differences in the $\mathrm{E}$ values between cubic and cylindrical models, and there were no significant differences in Von Mises stress distributions between the two models. These findings indicated that, to construct $\mu \mathrm{FE}$ models of vertebral trabecular bone, cubic or
\end{abstract}

Correspondence to: Professor Tian-Sheng Wang, Department of Orthopedics, 463 Hospital of PLA, 46 Xiaoheyan Road, Shenyang, Liaoning 110042, P.R. China

E-mail: wenxinxin1989@126.com

Professor Wei Lei, Department of Orthopedics, Xijing Hospital, Fourth Military Medical University, 15 Changle West Road, Xi'an, Shaanxi 710032, P.R. China

E-mail: leiwei@fmmu.edu.cn

*Contributed equally

Key words: micro-finite element model, trabecular bone, biomechanics, shape of model, human cylindrical models were both feasible. Choosing between the cubic or cylindrical $\mu \mathrm{FE}$ model is dependent upon the specific study design.

\section{Introduction}

Micro-finite element $(\mu \mathrm{FE})$ models, created from high resolution micro-computed tomography $(\mu-\mathrm{CT})$ images, have become a major computational tool for the assessment of the mechanical properties of human trabecular bone. By simulating a loading condition, this model then can be used to simulate the mechanical behavior of trabecular bone $(1,2)$, and has shown excellent prediction power compared with experimental measurements $(3,4) . \mu \mathrm{FE}$ analysis, based on this model, is potentially useful when evaluating the effects of bone diseases and their subsequent treatment on the mechanical properties of trabecular bone.

To date, $\mu \mathrm{FE}$ models of trabecular bone have predominantly been based on cored samples extracted from sites with high concentrations of the trabecular bone, for example, the vertebral body or proximal part of femur $(5,6)$. Among the studies using $\mu \mathrm{FE}$ models, the shapes of $\mu \mathrm{FE}$ models are typically cubical and cylinder. Gross et al (6) constructed cubic $\mu \mathrm{FE}$ models within the vertebral body in their study to investigate morphology-elasticity relationships. Cubic $\mu \mathrm{FE}$ models with a side length of $4 \mathrm{~mm}$ within the vertebral body were set to obtain the biomechanical properties of human trabecular bone (7). Cylindrical trabecular $\mu \mathrm{FE}$ models were also constructed to investigate the mechanical properties of vertebral trabecular bone $(3,8)$.

To the best of our knowledge, whether there are differences between cubic and cylindrical $\mu \mathrm{FE}$ models has not yet been studied. Therefore, the purpose of our study was to investigate the influence of the shape of the $\mu \mathrm{FE}$ model on the mechanical properties calculated from $\mu \mathrm{FE}$ analysis, and to determine whether there were differences between cubic and cylindrical $\mu \mathrm{FE}$ models. 


\section{Materials and methods}

Specimens preparation. In total, 5 lumbar vertebral bodies (L1-5) were collected form one embalmed human cadaver (61 years old; male) provided by the Department of Human Anatomy at the Fourth Military Medical University (Xi'an, China). Written informed consent from the donor was obtained for the use of these specimens in research. Collection and preparation procedures were approved by the Ethics Committee of the Fourth Military Medical University. All specimens were physically evaluated and radiographed to exclude bone diseases, bone cancers, and previous fractures. Soft tissues were carefully removed from the bone with a scalpel. Lateral and posterior elements including the pedicle, transverse process, and spinous process were excised from the vertebral body using a band saw (Isomet 1000, Buehler, Plymouth, MN, USA).

$\mu$-CT scanning. $\mu$-CT scans of all samples (5 vertebral bodies) were performed with a high-resolution $\mu$-CT system (Siemens Inveon; Siemens AG, Munich, Germany) with an isotropic resolution of $33.355 \mu \mathrm{m}$. Image processing included the application of a modest Gauss global filter and segmentation according to the method described by Otsu (9), which is a popular and established method in the field of threshold segmentation. Images were obtained using the following parameters: i) X-ray tube voltage, $100 \mathrm{kV}$; ii) anode current, $100 \mu \mathrm{A}$; and iii) shutter speed, 2,500 msec. High resolution images were obtained to produce $\mu \mathrm{FE}$ models for subsequent studies.

$\mu F E$ model building. $\mu \mathrm{FE}$ models were generated directly from the segmented images using a voxel conversion process (10). Firstly, all the DICOM image files were imported into the ScanIP software package (version 3.2; Build 1, Simpleware Ltd., Exeter, UK) to crop the different volumes of interest (VOI). As shown in Fig. 1, cubic cores (V1 in Fig. 1) of $8 \times 8 \times 8 \mathrm{~mm}^{3}$ were cropped to build cubic $\mu \mathrm{FE}$ models. Inscribed cylindrical cores (V2 in Fig. 1) of 8-mm diameter and 8-mm height were cropped to build cylindrical $\mu \mathrm{FE}$ models. Images were then segmented with the optimal threshold to match the bone volume fraction measured from $\mu$-CT analysis. The Floodfill function in the ScanIP software was used to remove all the floating or disconnected structures. Two layers of voxel elements were added at the superior and inferior surface to mimic stainless steel layers in axial compression tests.

Subsequently, the files were imported into ScanFE (version 3.1.2, Build 2, Simpleware Ltd., Exeter, UK) to construct the $\mu \mathrm{FE}$ model. With this approach, voxels representing bone tissue were converted to equally sized eight-node hexahedral voxel elements, whereas voxels representing the bone marrow were ignored. All the models were imported to ANSYS (release 14.0; ANSYS, Inc., Township, PA, USA) to perform further calculations. For all models, the element material properties of bone were considered to be isotropic, linear elastic, and uniform with a tissue Young's modulus of $10 \mathrm{GPa}$ and a tissue Poisson's ratio of 0.3 (11). An isotropic homogenous tissue modulus of $200 \mathrm{GPa}$ and a tissue Poisson's ratio of 0.3 was assigned to the element of stainless steel layers (12).

Computational process of $\mu F E$ analysis. A $1 \%$ axial strain was prescribed to the top surface of the model, and axial

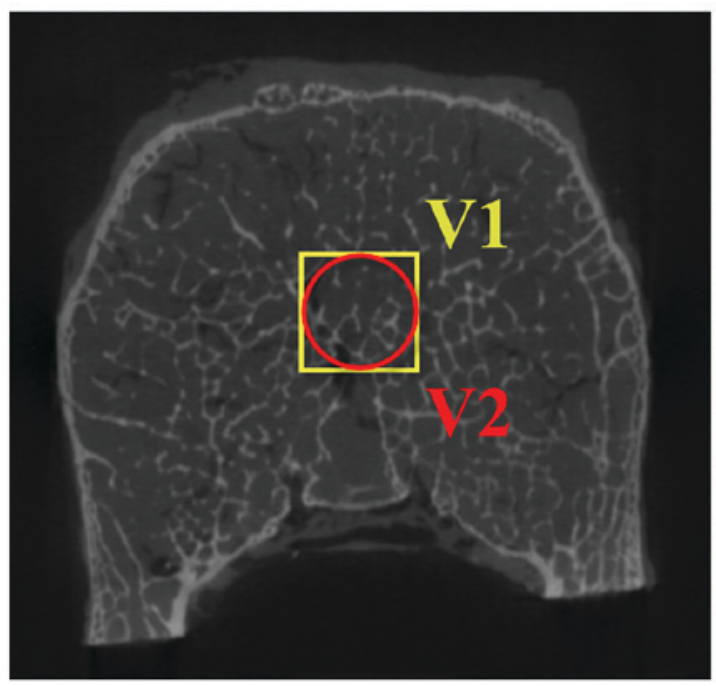

Figure 1. VOI in the vertebral body. Cubic VOI is shown as V1 in yellow color, and inscribed cylindrical VOI is shown as V2 in red color. VOI, volume of interest.

displacements at the bottom surface were constrained, simulating an axial compression test along the superior-inferior direction. Contact between the upper and lower surfaces of the specimen and the steel plates were modeled using contact elements with a zero friction value to ensure that only compressive forces were transmitted (13). All analyses were performed on a workstation computer (ThinkStation; Intel Xeon CPU E3-122, 3.10 GHz, Lenovo Group Ltd., Beijing, China). The apparent stresses were calculated as the total reaction force per apparent area. Based on that, the E values were calculated by dividing the apparent strain by 0.01 (14).

Statistical analysis. Statistical analyses were performed using the SigmaPlot 12.5 (Systat Software Inc., San Jose, CA, USA). Paired-t tests were performed to determine whether there were differences between the $\mathrm{E}$ values obtained from cubic and cylindrical models. $\mathrm{P}<0.05$ was considered to indicate a statistically significant difference.

\section{Results}

Von Mises stress distributions. Von Mises stress distributions in the trabecular bone structure of cubic and cylindrical $\mu \mathrm{FE}$ models are shown in Fig. 2. There were no significant differences of Von Mises stress distributions between cubic and cylindrical $\mu \mathrm{FE}$ models.

$E$ values. E values obtained from $\mu \mathrm{FE}$ analyses are shown in Fig. 3. E of the cubic models was $146.34 \pm 9.76 \mathrm{MPa}$, and of cylindrical models was 139.35 $\pm 13.21 \mathrm{MPa}$. Paired t-tests showed that there were no statistically significant differences in $\mathrm{E}$ values between the cubic and cylindrical models.

\section{Discussion}

In the present study, cubic and cylindrical $\mu \mathrm{FE}$ models were built to investigate if there were significant differences between these two models. No significant differences in the $\mathrm{E}$ 

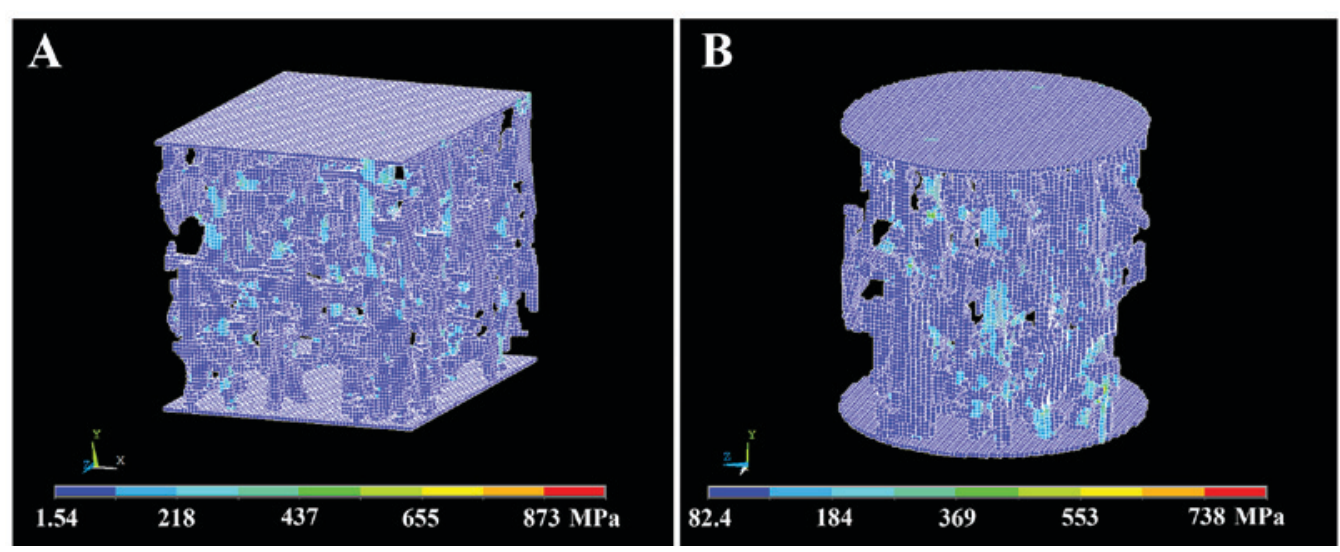

Figure 2. Von Mises stress distribution in the trabecular bone structure of cubic and cylindrical $\mu \mathrm{FE}$ models. (A) Cubic $\mu \mathrm{FE}$ models and (B) cylindrical $\mu \mathrm{FE}$ models. $\mu \mathrm{FE}$, micro-finite element.

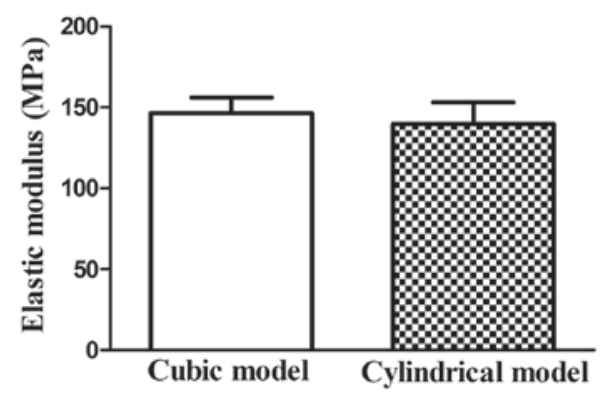

Figure 3. E values calculated from cubic and cylindrical models. Paired t-test demonstrated that there were no significant differences between these two models. Data are presented as the mean \pm standard deviation.

values were detected between the cubic and cylindrical $\mu \mathrm{FE}$ models.

$\mu \mathrm{FE}$ analysis is now widely used to investigate bone mechanical properties; these mechanical properties have been demonstrated to relate to bone microarchitecture (15). Based on high resolution 3D images obtained from $\mu \mathrm{CT}, \mu \mathrm{FE}$ models were constructed for further simulation calculations. By simulating a loading condition, this model can be used to derive the elastic modulus of the bone, as well as the distribution of stresses and strains in the bone tissue $(1,16)$.

Obtained from $\mu \mathrm{FE}$ models, trabecular bone modulus has a good correlation with experimental modulus and strength $(15,17)$. Elastic modulus obtained in our studies were similar to former experimental compression studies on trabecular bone samples $(18,19)$. Compression tests on vertebral trabecular bone cores have shown that the apparent elastic modulus on axial direction was $189.7 \mathrm{MPa}$ (18), which is simular to the value of 139.96-146.34 MPa obtained in the present study. In another study based on vertebral trabecular bone, apparent elastic modulus calculated from $\mu \mathrm{FE}$ models was $146-154 \mathrm{MPa}$ (19). These findings indicated that the $\mu \mathrm{FE}$ models constructed in our study are reliable.

Voxels representing bone tissue were converted to equally sized eight-node hexahedral voxel elements to construct $\mu \mathrm{FE}$ models in our study. A number of studies have investigated the effect of voxel size on the accuracy of biomechanical measurements on human trabecular bone (20-22). Voxel size predominantly refers to scanning and reconstruction voxel size. Scanning voxel size is based on the $\mu$-CT scanning resolution. A previous study demonstrated that it is the resolution of raw data that primarily determines the accuracy of models as the bone volume fraction of bone volume/total volume was predominantly affected by the scanning resolution (22). The scanning resolution of the $\mu$-CT system used in our study was $33.355 \mu \mathrm{m}$, which was enough to depict the microarchitecture of human trabecular bone $(23,24)$. In another study, the recommended resolution in finite element models of trabecular bone was one quarter of trabecular thickness (25). The thickness of trabeculae in our study was $\sim 200 \mu \mathrm{m}$ and the resolution of $33.355 \mu \mathrm{m}$, fulfilling the conditions rule. Reconstruction voxel size is the actual voxel size concerted to the $3 \mathrm{D} \mu \mathrm{FE}$ model. The reconstruction voxel size in our study was identical with scanning voxel size, and this could avoid the inaccuracy of coarsening reconstruction voxel size.

The size of cubic and cylindrical models in our study has contained enough structural information for virtual compressions. Pahr and Zysset (26) suggested that the side length of the volume should be $>5 \mathrm{~mm}$ as it may provide the proposed boundary conditions. Another study showed that the VOI of trabecular bone should be $>6 \times 6 \times 6 \mathrm{~mm}^{3}$ to predict the microarchitecture of human trabecular bone (27). The VOI of trabecular bone in our study met these conditions. The $\mu \mathrm{FE}$ models constructed in our study included a representation of steel plate located on the upper and lower surfaces of the specimen. The lower steel plate was constrained and compressive displacement was applied to the upper steel plate. All these conditions stimulated the experimental compression test better.

Notably, the cubic and cylindrical $\mu \mathrm{FE}$ models were constructed from the same position of the vertebral body. It has been confirmed that the architecture of the trabecular bone within the vertebral body is inhomogeneous (17,28-31). Within the vertebral body, the trabecular architecture in the posterior region was superior to the anterior region $(28,31)$. Compared with the posterior region, the anterior region had lower bone mass density, less trabecular bone volume fraction, less trabecular number, and greater trabecular separation (28). Although there were no significant differences between cubic and cylindrical $\mu \mathrm{FE}$ models, the positions of the trabecular bone core should be identical. 
In experimental compression tests, trabecular bone cores extracted from vertebral bodies are often used $(23,32)$. In our study, cubic and cylindrical $\mu \mathrm{FE}$ models were constructed to investigate if there were differences between the two models. This indicated that the shape of trabecular bone core used in experimental biomechanical tests may not affect the biomechanical properties obtained from these real tests. These computational models used in our study may enable us to make repeated virtual compression tests on trabecular bone core extracted from the same specimen. This may avoid the influence of variance among different specimens and ensure the results are more reliable.

To construct $\mu \mathrm{FE}$ models of trabecular bone, choosing the VOI was critical. Cubic and cylindrical VOI are the most commonly used shapes of VOI $(3,33)$. The results demonstrated that there was no significant difference between these two models. With regard to the Von Mises stress distributions, there were also no significant differences between cubic and cylindrical $\mu \mathrm{FE}$ models. Choosing between the cubic or cylindrical models shall depend on the specific study design. A limitation of the present study was that the trabecular bone specimens used in our study were collected from vertebral bodies. It has is well-documented that the morphologies of trabecular bone harvested from different skeletal sites are quite different $(34,35)$. Further research is required to confirm that the findings of the present study are applicable to other skeletal sites.

In conclusion, to construct $\mu \mathrm{FE}$ models of vertebral trabecular bone, cubic or cylindrical models were both feasible. The present findings demonstrated that there were no significant difference between these two shapes of models. To choose cubic or cylindrical model, it depends on the specific study design.

\section{Acknowledgements}

The authors would like to thank Jia-Ji Yang at the School of Stomatology of Fourth Military Medical University for their technical assistance at $\mu \mathrm{CT}$ analysis. This study was supported by the National Nature Science Foundation of China (grant no. 81301292).

\section{References}

1. Niebur GL, Feldstein MJ, Yuen JC, Chen TJ and Keaveny TM: High-resolution finite element models with tissue strength asymmetry accurately predict failure of trabecular bone. J Biomech 33 : 1575-1583, 2000.

2. Verhulp E, Van Rietbergen B, Muller R and Huiskes R: Micro-finite element simulation of trabecular-bone post-yield behaviour-effects of material model, element size and type. Comput Methods Biomech Biomed Engin 11: 389-395, 2008.

3. Wolfram U, Wilke HJ and Zysset PK: Valid micro finite element models of vertebral trabecular bone can be obtained using tissue properties measured with nanoindentation under wet conditions. J Biomech 43: 1731-1737, 2010.

4. Chevalier Y,PahrD, Allmer H,Charlebois MandZyssetP: Validation of a voxel-based FE method for prediction of the uniaxial apparent modulus of human trabecular bone using macroscopic mechanical tests and nanoindentation. J Biomech 40: 3333-3340, 2007.

5. Hambli R: Apparent damage accumulation in cancellous bone using neural networks. J Mech Behav Biomed Mater 4: 868-878, 2011.

6. Gross T, Pahr DH and Zysset PK: Morphology-elasticity relationships using decreasing fabric information of human trabecular bone from three major anatomical locations. Biomech Model Mechanobiol 12: 793-800, 2013.
7. Ulrich D, van Rietbergen B, Laib A and Rüegsegger P: The ability of three-dimensional structural indices to reflect mechanical aspects of trabecular bone. Bone 25: 55-60, 1999.

8. Bevill G, Eswaran SK, Farahmand F and Keaveny TM: The influence of boundary conditions and loading mode on high-resolution finite element-computed trabecular tissue properties. Bone 44: 573-578, 2009.

9. Otsu N: A threshold selection method from gray-level histograms. IEEE Trans Syst Man Cybern 9: 62-66, 1979.

10. Ruffoni D, Wirth AJ, Steiner JA, Parkinson IH, Müller R and van Lenthe GH: The different contributions of cortical and trabecular bone to implant anchorage in a human vertebra. Bone 50: 733-738, 2012.

11. Ulrich D, van Rietbergen B, Weinans $H$ and Rüegsegger P: Finite element analysis of trabecular bone structure: A comparison of image-based meshing techniques. J Biomech 31: 1187-1192, 1998

12. Kamaya M: Stress-strain curve estimation procedures for stainless steels based on yield and ultimate strengths. Eng Fract Mech 127: 194-210, 2014

13. Hambli R: Micro-CT finite element model and experimental validation of trabecular bone damage and fracture. Bone 56: 363-374, 2013.

14. Cowin SC: Bone mechanics handbook. 2nd edition. Boca Raton, CRC Press, 2001

15. Hou FJ, Lang SM, Hoshaw SJ, Reimann DA and Fyhrie DP: Human vertebral body apparent and hard tissue stiffness. J Biomech 31: 1009-1015, 1998.

16. van Rietbergen B and Ito K: A survey of micro-finite element analysis for clinical assessment of bone strength: The first decade. J Biomech 48: 832-841, 2015.

17. Kim DG, Hunt CA, Zauel R, Fyhrie DP and Yeni YN: The effect of regional variations of the trabecular bone properties on the compressive strength of human vertebral bodies. Ann Biomed Eng 35: 1907-1913, 2007.

18. Aiyangar AK, Vivanco J, Au AG, Anderson PA, Smith EL and Ploeg HL: Dependence of anisotropy of human lumbar vertebral trabecular bone on quantitative computed tomography-based apparent density. J Biomech Eng 136: 091003, 2014.

19. Depalle B, Chapurlat R, Walter-Le-Berre H, Bou-Saïd B and Follet H: Finite element dependence of stress evaluation for human trabecular bone. J Mech Behav Biomed Mater 18: 200-212, 2013.

20. Kim DG, Christopherson GT, Dong XN, Fyhrie DP and Yeni YN: The effect of microcomputed tomography scanning and reconstruction voxel size on the accuracy of stereological measurements in human cancellous bone. Bone 35: 1375-1382, 2004.

21. Maloul A, Fialkov J and Whyne C: The impact of voxel size-based inaccuracies on the mechanical behavior of thin bone structures. Ann Biomed Eng 39: 1092-1100, 2011.

22. Yeni YN, Christopherson GT, Dong XN, Kim DG and Fyhrie DP: Effect of microcomputed tomography voxel size on the finite element model accuracy for human cancellous bone. J Biomech Eng 127: 1-8, 2005.

23. Bauer JS, Sidorenko I, Mueller D, Baum T, Issever AS, Eckstein F, Rummeny EJ, Link TM and Raeth CW: Prediction of bone strength by $\mu \mathrm{CT}$ and MDCT-based finite-element-models: How much spatial resolution is needed? Eur J Radiol 83: e36-e 42, 2014.

24. Isaksson H, Töyräs J, Hakulinen M, Aula AS, Tamminen I, Julkunen P, Kröger H and Jurvelin JS: Structural parameters of normal and osteoporotic human trabecular bone are affected differently by microCT image resolution. Osteoporos Int 22: 167-177, 2011.

25. Niebur GL, Yuen JC, Hsia AC and Keaveny TM: Convergence behavior of high-resolution finite element models of trabecular bone. J Biomech Eng 121: 629-635, 1999.

26. Pahr DH and Zysset PK: Influence of boundary conditions on computed apparent elastic properties of cancellous bone. Biomech Model Mechanobiol 7: 463-476, 2008.

27. Yan YB, Qi W, Wang J, Liu LF, Teo EC, Tianxia Q, Ba JJ and Lei W: Relationship between architectural parameters and sample volume of human cancellous bone in micro-CT scanning. Med Eng Phys 33: 764-769, 2011.

28. Wang Y, Owoc JS, Boyd SK, Videman T and Battié MC: Regional variations in trabecular architecture of the lumbar vertebra: Associations with age, disc degeneration and disc space narrowing. Bone 56: 249-254, 2013.

29. Wegrzyn J, Roux JP, Arlot ME, Boutroy S, Vilayphiou N, Guyen O, Delmas PD, Chapurlat R and Bouxsein ML: Role of trabecular microarchitecture and its heterogeneity parameters in the mechanical behavior of ex vivo human L3 vertebrae. J Bone Miner Res 25: 2324-2331, 2010. 
30. Chen H, Shoumura S, Emura S and Bunai Y: Regional variations of vertebral trabecular bone microstructure with age and gender. Osteoporos Int 19: 1473-1483, 2008.

31. Hulme PA, Boyd SK and Ferguson SJ: Regional variation in vertebral bone morphology and its contribution to vertebral fracture strength. Bone 41: 946-957, 2007.

32. Parkinson IH, Badiei A, Stauber M, Codrington J, Müller R and Fazzalari NL: Vertebral body bone strength: The contribution of individual trabecular element morphology. Osteoporos Int 23: 1957-1965, 2012

33. Lü L, Meng G, Gong H, Zhu D, Gao J and Fan Y: Tissue level microstructure and mechanical properties of the femoral head in the proximal femur of fracture patients. Acta Mech Sinica 31: 259-267, 2015
34. Hildebrand T, Laib A, Müller R, Dequeker J and Rüegsegger P: Direct three-dimensional morphometric analysis of human cancellous bone: Microstructural data from spine, femur, iliac crest, and calcaneus. J Bone Miner Res 14: 1167-1174, 1999.

35. Eckstein F, Matsuura M, Kuhn V, Priemel M, Müller R, Link TM and Lochmüller EM: Sex differences of human trabecular bone microstructure in aging are site-dependent. J Bone Miner Res 22: 817-824, 2007. 